\title{
Is there a critical LH level for hCG trigger after the detection of LH surge in modified natural frozen-thawed single blastocyst transfer cycles?
}

\author{
Semra Kahraman ${ }^{1}$ (1) $\cdot$ Yucel Sahin ${ }^{1}$ \\ Received: 23 May 2020 / Accepted: 7 October 2020 / Published online: 14 October 2020 \\ (C) The Author(s) 2020
}

\begin{abstract}
Purpose There is no consensus yet in the literature on an optimal luteinizing hormone (LH) level for human chorionic gonadotrophin (hCG) trigger timing in patients undergoing frozen-thawed embryo transfer (FET) with modified natural cycles (mNC). The objective of our study was to compare the clinical results of hCG trigger at different LH levels in mNC-FET cases.

Methods This retrospective study was conducted in Istanbul Memorial Hospital ART and Genetics Center. A total of 1076 cases with $1163 \mathrm{mNC}-\mathrm{FET}$ cycles were evaluated. LH levels between the start of LH rise (15 IU/L) and LH peak level (> $40 \mathrm{IU} / \mathrm{L})$ were evaluated. Cycles were analyzed in four groups: group A $(n=287) \mathrm{LH}$ level on the day prior to the day of hCG; groups $\mathrm{B}, \mathrm{C}$ and D, LH levels on the day of hCG: group B $(n=245)$ LH 15-24.9; group C ( $n=253)$, LH 25-39.9; group D $(n=383)$ LH $\geq 40$. Cycle outcomes in the four groups were compared.

Results Subgroup analyses of mNC-FET groups showed that implantation, clinical and ongoing pregnancy rates, and pregnancy losses were not significantly different in patients with different LH levels on the day of hCG trigger.

Conclusion Our study suggests that hCG can be administered at any time between the start of LH rise ( $\geq 15 \mathrm{IU} / \mathrm{L})$ and LH peak level ( $\geq 40 \mathrm{IU} / \mathrm{L})$ without a detrimental effect on clinical outcome.
\end{abstract}

Keywords $\mathrm{mNC}$-FET $\cdot \mathrm{LH}$ value $\cdot$ hCG day

\section{Introduction}

Advances in cryopreservation techniques have resulted in a dramatic increase in freeze-all cycles. Reasons for this preference include the higher rate of viable embryos available after thawing and the near elimination of the risk of ovarian hyperstimulation syndrome (OHSS). Furthermore, many studies have shown increased implantation rates when transfer takes place in a more natural uterine environment without elevated levels of hormones [1-4].

Appropriate endometrial preparation therefore plays an essential role in successful implantation in FET cycles. The two main alternative approaches for endometrial preparation are

Semra Kahraman

semkahraman@gmail.com

1 Assisted Reproductive Technologies and Reproductive Genetics Center, Istanbul Memorial Hospital, Piyalepasa Bulvari, Sisli, 34384 Istanbul, Turkey estrogen and progesterone replacement treatment (artificial cycle) or the use of natural cycle, depending on the regularity or pattern of the menstrual cycle. Because of possible adverse hormonal effects, including a higher risk of thrombo-embolic events, and because of the inconvenience to patients, in our center, the artificial cycle is used only for cases with anovulatory cycles and/or endometrial thinning.

In natural cycle, the alternatives are true and modified natural cycles. True natural cycle (tNC)-FET requires strict hormonal and follicular development monitoring. On the other hand, the mNC-FET has a number of advantages. Firstly, continual hormonal monitoring is not required. Secondly, once the hCG trigger is performed, the timing of embryo transfer can be determined. Thirdly and importantly, hCG injection not only triggers ovulation but also supports the luteal phase. Because of these advantages, mNC-FET is the preferred technique in our center. However, the results of studies focusing on LH level at the time of hCG in mNC-FET cycle have been variable and there is no consensus yet on the optimal LH level for hCG trigger timing. 
There are several papers examining the optimal identification of LH surge and peak and the determination of embryo transfer day. Irani et al. [5] showed that in true natural cycle, defining the surge as occurring on the day of an LH of $\geq 17 \mathrm{IU} /$ L with a drop of $\geq 30 \%$ in estradiol (E2) levels, the following day was associated with better NC-FET outcomes than defining the surge as the day representing the highest serum $\mathrm{LH}$ level with $\mathrm{a} \geq 30 \%$ drop in E2 levels on the same day [5]. A further related study by Reichman et al. [6], in which the LH surge was defined by an LH level $>17 \mathrm{IU} / \mathrm{L}$ during the follicular phase with a subsequent dropping E2 level thereafter, also reported improved cycle outcomes with hCG booster given to patients within 1 day post-LH surge. Bartels et al. [7] reported that when $\mathrm{LH}$ reached $\geq 20 \mathrm{IU} / \mathrm{L}$, there was no difference in outcomes regardless of whether transfer was performed on day 6 or day 7 after the initial surge. On the other hand, Litwicka et al. [8] suggested that LH elevation $\geq 13$ before hCG administration may negatively affect the clinical results in $\mathrm{mNC}$ and advised routine performance of $\mathrm{LH}$ determination during the regular ultrasonographic evaluation of dominant follicle growth and avoidance of hCG in cases of elevated LH, when embryo transfer should be performed after spontaneous follicular rupture. Kyrou et al. [9] reported higher ongoing pregnancy rates in patients with spontaneous LH surge compared with hCG-administered patients undergoing natural cycle intrauterine insemination. They suggested that ovulation and implantation are driven primarily by the $\mathrm{LH}$ surge itself and only to a lesser extent by exogenous hCG [9]. Another study by Fatemi et al. [10] concluded that there is emerging evidence that the administration of hCG in the late follicular phase induces a cascade of events in the endometrium, which would have started several days later in the presence of endogenous LH rise and that these events appear to have a negative impact on ongoing pregnancy rate.

Our retrospective study, based on $1168 \mathrm{mNC}-\mathrm{FET}$ cycles in good prognosis patients only, investigates whether there is a critical LH level for hCG trigger after the detection of LH surge in modified natural frozen-thawed single blastocyst transfer cycles.

\section{Material and methods}

\section{Patient population}

This retrospective study, conducted between January 2015 and September 2019 in Istanbul Memorial Hospital ART and Genetics Center was based on $1168 \mathrm{mNC}$-FET cycles in 1076 cases with regular menses and evidence of ovulation. Patients with regular menses (24-35 days) for at least the last 6 months and with ovulatory cycles proven with ultrasonography or with hormonal evaluation and who were undergoing frozen-thawed single blastocyst transfer cycles were evaluated. Inclusion criteria were: $\leq 42$ years old, body mass index (BMI) $<30 \mathrm{~kg} / \mathrm{m}^{2}$, blastocyst-stage frozen-thawed single embryo transfer on day 5 , or only rarely on day $6(n=12)$, endometrial thickness of $\geq 8 \mathrm{~mm}$. Cases with recurrent pregnancy losses $(\geq 3)$ and repeated implantation failure ( $>3$ cycles) were excluded. Patients undergoing PGT-A were not included in this study. This study was approved by Istanbul Memorial Hospital institutional review board (Number: 2020/004).

Our aim was to compare single blastocyst transfer outcomes, therefore a good prognosis group was chosen for the study, because elective blastocyst-stage single embryo transfer is more likely to be possible. Furthermore, including only good prognosis patients excluded any possible bias which could have resulted from compromised clinical characteristics of patients. In cases with elevated serum progesterone $(\geq 3 \mathrm{ng} / \mathrm{ml}$ ) and/or the formation of corpus luteum, the cycle was continued as a true natural cycle and therefore not included in our study.

The following data were recorded for each patient: the causes of infertility (male factor, unexplained infertility, tubal factor, endometriosis, diminished ovarian reserve (DOR) and combined factors), maternal age, BMI, and anti-Mullerian hormone (AMH) level. Fresh cycle characteristics were evaluated, including mean number of cumulus oocyte complex (COC), metaphase II (MII) oocytes and fertilized oocytes, and blastocyst morphology (the percentage of excellent, good, moderate, poor).

\section{Follicular monitoring and endometrial preparation}

Ultrasonographic evaluation was carried out on the second day of menstruation to rule out the presence of ovarian cysts or other pelvic pathologies. It was repeated on day 9 or 10 , depending on the cycle length, to observe whether there was a spontaneously growing dominant follicle. After day 9 or 10 , it was repeated every 2 or 3 days to monitor endometrial thickness and follicular size. When the follicle reached $15 \mathrm{~mm}$, serum LH and estrogen were evaluated and levels were measured every $24 \mathrm{~h}$ until detecting the LH surge.

The duration from the first day of menstrual cycle to the hCG trigger day and then to the embryo transfer day, follicular diameter on hCG day, endometrial thickness on hCG day, E2 level on hCG day were recorded. Definitions of the beginning of LH surge vary between $\geq 10 \mathrm{IU} / \mathrm{L}$ and $\geq 20 \mathrm{IU} / \mathrm{L}$ in different studies [6, 7, 11-13]. In our study LH surge was defined as the LH level reaching $\geq 15 \mathrm{IU} / \mathrm{L}$ and $\mathrm{LH}$ peak as the $\mathrm{LH}$ level reaching $\geq 40 \mathrm{IU} / \mathrm{L}$. This was determined by evaluating data based on the strict monitoring of estrogen, $\mathrm{LH}$, and progesterone levels of true natural cycle cases in our clinic over a period of 8 years.

mNC-FET cycles were evaluated retrospectively and grouped according to LH levels. LH levels on the day of 
hCG were the main focus of the study. In groups B, C and D, LH levels on the day of hCG were: group B $(n=245)$ LH 15$24.9 \mathrm{IU} / \mathrm{L}$; group C $(n=253)$, LH 25-39.9 IU/L; group D ( $n=$ 383) $\mathrm{LH} \geq 40 \mathrm{IU} / \mathrm{L}$. In these groups, recombinant hCG (rhCG) (Ovitrelle ${ }^{\circledR}$, Merck Serono, Switzerland) was administered at any time between the start of LH rise (15 IU/L) and LH peak level $(\geq 40 \mathrm{IU} / \mathrm{L})$. rhCG was given in the evening in all groups and frozen embryo transfer was scheduled for 6 days after rhCG administration.

When our data was evaluated retrospectively, we identified a fourth group (group A, $n=287$ ) who lived at significant distances from the hospital and for whom next day clinic attendance was not practical. Ultrasonographic evaluations had been continued until their final appointment at our clinic, when their LH levels (mean: $14.8 \mathrm{IU} / \mathrm{L}$ ) indicated that $\mathrm{LH}$ rise had already started or would start the next day. Therefore, one more day was allowed either for the follicle to increase in size or for the LH level to rise. They were advised to administer the hCG trigger in the evening of the next day instead of returning to the hospital. Therefore, in this group, the LH level on the day prior to the day of hCG was recorded. The findings regarding this group are included in order to illustrate practical approaches for the clinician.

Frozen embryo transfer was scheduled for 5 or rarely 6 days after rhCG administration. Vaginal progesterone gel with $90 \mathrm{mg}(8 \%)$ (Crinone ${ }^{\circledR}$ Merck Serono, Switzerland) administered once a day was started two days after rhCG administration to reduce the risk of luteal phase deficiency and poor endogenous luteal phase. When pregnancy occurred, the same daily doses of progesterone vaginal gel were continued until the 10 th week of gestation.

\section{Embryo scoring, vitrification, and thawing}

Blastocysts were scored before vitrification according to Gardner's classification [14]. Good or top-quality blastocysts (at least 3BB) were vitrified on day 5 and day 6 morning with Kitazato vitrification media, using Cryotops ${ }^{\circledR}$ as carriers. Blastocysts were thawed with Kitazato warming media according to manufacturer's instructions. Embryos were first checked for survival $30 \mathrm{~min}$ after thawing. A second check occurred $2 \mathrm{~h}$ after warming for re-expansion, hatching, extensive cytoplasmic granulation, and the presence of necrotic foci, which are predictors of the rates of implantation, pregnancy, and live birth [7]. Eligible blastocysts with at least $80 \%$ re-expansion and vitality were transferred in the afternoon of the same day.

\section{Evaluation of clinical outcomes}

Nine days after blastocyst transfer, serum $\beta$-hCG was measured. Clinical pregnancy was diagnosed if gestational sac was observed, viable pregnancy was defined as the presence of fetal heart beat at week 7 and ongoing pregnancy was defined as a 12 -week viable pregnancy.

All pregnancy rates including biochemical pregnancy rate (BPR), clinical pregnancy rate (CPR), and ongoing pregnancy rate (OPR) were calculated according to per embryo transfer cycle. BPR was defined as the number of biochemical pregnancies/number of ET cycles, CPR as the number of clinical pregnancies/number of ET cycles and OPR as the number of ongoing pregnancies/number of ET cycles.

Total pregnancy loses (TPL) was defined as pregnancies resulting in first trimester losses. Biochemical pregnancy loss (BPL) was defined as pregnancy resulting in loss before sonographically visible gestational sac formation and clinical pregnancy loss (CPL) was defined as pregnancy loss after gestational sac formation and before 12-week pregnancy.

The rate of biochemical pregnancy losses (BPLR) was defined as the number of BPL/number of biochemical pregnancies. The rate of clinical pregnancy losses (CPLR) was defined as the number of CPL/number of clinical pregnancies. The rate of total pregnancy losses (TPLR) was defined as the number of TPL/number of biochemical pregnancies.

\section{Statistics}

In our study, the total number of cases needed to obtain $80 \%$ power at $\alpha=0.03$ level was found to be $n=787$ cycles. However, to take account of possible losses, it was considered that a cycle number of approximately 1000 would be more reliable.

For statistical analyses NCSS (Number Cruncher Statistical System) 2007 Statistical Software (Utah, USA) program was used. Demographics of patients were reported as minimum, maximum, mean $\pm \mathrm{SD}$ and $95 \%$ confidence interval of the mean. In addition, Kolmogorov Smirnov test and box plot charts were used to compare the data to normal distribution. As a result of these tests, it was determined that data showed a normal distribution for all variables. One-way ANOVA test with Bonferroni correction for multiple comparisons was applied for continuous variables and Pearson chi-square test for categorical group comparisons. A $p$ value of $p<0.05$ was accepted as statistically significant.

\section{Results}

A total of 1076 cases with $1168 \mathrm{mNC}$-FET cycles were retrospectively evaluated.

Table 1 shows patient characteristics according to $\mathrm{LH}$ levels. There was no statistically significant difference between the LH groups in terms of demographic characteristics or infertility diagnosis. Bonferroni correction showed that the only significant differences occurred between group $\mathrm{D}$ and group B in terms of cycle lengths to hCG day $(p=0.027)$ 
Table 1 Patient and FET cycle characteristics according to LH levels

\begin{tabular}{|c|c|c|c|c|c|}
\hline Characteristics & $\begin{array}{l}\text { Group A } \\
(n=287)\end{array}$ & $\begin{array}{l}\text { Group B } \\
(n=245)\end{array}$ & $\begin{array}{l}\text { Group C } \\
(n=253)\end{array}$ & $\begin{array}{l}\text { Group D } \\
(n=383)\end{array}$ & $p$ value \\
\hline Age (years) $($ mean $\pm \mathrm{SD})$ & $32.87 \pm 4.96$ & $32.43 \pm 4.82$ & $32.4 \pm 4.71$ & $32.18 \pm 4.81$ & ${ }^{\mathrm{a}} 0.452$ \\
\hline BMI $\left(\mathrm{kg} / \mathrm{m}^{2}\right)($ mean $\pm \mathrm{SD})$ & $23.99 \pm 3.98$ & $23.92 \pm 4.02$ & $24.42 \pm 4.28$ & $24.13 \pm 4.01$ & ${ }^{\mathrm{a}} 0.639$ \\
\hline $\mathrm{AMH}(\mathrm{ng} / \mathrm{ml})(\mathrm{mean} \pm \mathrm{SD})$ & $3.11 \pm 2.80$ & $3.19 \pm 2.55$ & $3.16 \pm 2.33$ & $3.01 \pm 2.36$ & ${ }^{\mathrm{a}} 0.896$ \\
\hline Cycle length up to hCG day $(\mathrm{d})($ mean \pm SD) & $13.05 \pm 2.80$ & $11.69 \pm 2.17$ & $13.16 \pm 2.66$ & $13.84 \pm 2.95$ & ${ }^{\mathrm{a}} 0.027 *$ \\
\hline Cycle length up to ET day (d) (mean \pm SD) & $19.08 \pm 3.39$ & $18.07 \pm 2.95$ & $19.11 \pm 3.05$ & $19.74 \pm 3.44$ & ${ }^{\mathrm{a}} 0.033^{*}$ \\
\hline Peak endometrial thickness $(\mathrm{mm})($ mean $\pm \mathrm{SD})$ & $10.59 \pm 1.70$ & $10.73 \pm 1.79$ & $10.65 \pm 1.84$ & $10.89 \pm 1.71$ & ${ }^{\mathrm{a}} 0.055$ \\
\hline E2 Level on hCG day $(\mathrm{pg} / \mathrm{ml})($ mean $\pm \mathrm{SD})$ & $238.28 \pm 105$ & $295.1 \pm 100.5$ & $287.3 \pm 89.6$ & $255 \pm 84.8$ & ${ }^{\mathrm{a}} 0.055$ \\
\hline $\begin{array}{l}\text { LH level on the day before hCG (group A) and on the day } \\
\text { of hCG (groups B, C, and D) (IU/L) (mean } \pm \text { SD) }\end{array}$ & $14.8 \pm 15.4$ & $20.2 \pm 12.8$ & $32.1 \pm 14.2$ & $61.7 \pm 18.9$ & ${ }^{\mathrm{a}} 0.01 *$ \\
\hline Follicle size $(\mathrm{mm})($ mean $\pm \mathrm{SD})$ & $18.3 \pm 1.5$ & $18.1 \pm 1.4$ & $18.4 \pm 1.6$ & $18.3 \pm 1.5$ & ${ }^{\mathrm{a}} 0.113$ \\
\hline Infertility factor & & & & & b 0.465 \\
\hline Male factor & $72(25.1)$ & $64(26.1)$ & $69(27.3)$ & $99(25.8)$ & \\
\hline Unexplained & $61(21.2)$ & $49(20.1)$ & $47(18.6)$ & $82(21.4)$ & \\
\hline Tubal factor & $52(18.2)$ & $40(16.3)$ & $40(15.7)$ & $63(16.4)$ & \\
\hline Endometriosis & $6(2.1)$ & $4(1.6)$ & $7(2.8)$ & $6(1.6)$ & \\
\hline DOR & $44(15.3)$ & $45(18.4)$ & $43(17)$ & $59(15.4)$ & \\
\hline Combined factor & $52(18.1)$ & $43(17.5)$ & $47(18.6)$ & 74 (19.4) & \\
\hline
\end{tabular}

${ }^{\mathrm{a}}$ One-way ANOVA

${ }^{\mathrm{b}}$ Pearson chi-square test

$* p<0.05$

The differences between the groups were compared using the Bonferroni correction. Cycle length from the first day of menstruation to hCG day is significantly longer in group D than in group B $(p=0.031)$ and also cycle length from the first day of menstruation to ET day is significantly longer in group D than in group B $(p=0.048)$

and to embryo transfer day $(p=0.033)$. Cycle length from the first day of menstruation to hCG day was significantly longer in group D than in group B $(p=0.031)$ and also cycle length from the first day of menstruation to ET day was significantly longer in group D than in group B $(p=0.048)$.

Table 2 summarizes the fresh cycle characteristics. No significant difference was observed between the groups.

Table 3 shows pregnancy outcomes. No significant difference was observed between different LH groups in BPR, CPR, and OPRs. In addition, no significant difference was observed in BPLR, CPLR and TPLR between different LH groups.

\section{Discussion}

Artificial endometrial preparation with hormones and natural cycle are the most commonly used endometrial preparation techniques [15]. The most effective, practical, convenient and safe endometrial preparation protocol is still under debate. The natural cycle may be preferable for women with regular menstrual cycles as, being more physiological, it requires less medication [16]. tNC-FET requires strict hormonal and follicular development monitoring. mNC-FET, which requires administration of exogenous hCG, has the advantage of supporting the luteal phase.

Transfer of the thawed embryo should be conducted at the time of optimal endometrial receptivity, which is determined by the time of LH surge during tNC-FET. In the $\mathrm{mNC}$, in order to determine the timing of the hCG administration some studies report using follicular size only whereas others use both follicular size and the detection of LH surge [17-19]. Currently however, there is no consensus on the exact parameters that define the LH surge.

Groenewoud et al. [17] found no optimal cut-off LH concentration and argued that regular ultrasound evaluation of the dominant follicle in combination with hCG triggering of ovulation was an effective way of FET planning. They concluded that single LH determination prior to ovulation induction in unstimulated cycle FET does not seem to have added clinical value [17]. However, Saupstad et al. [20] reported that in a natural cycle the follicle size corresponding to the start of $\mathrm{LH}$ rise varies between different patients. Therefore, relying on follicular size only may result in the administration of hCG either too early or too late, consequently adversely affecting endometrial receptivity, thereby resulting in asynchronicity between the endometrium and the transferred blastocyst. In our study, the timing of hCG was determined not only by 
Table 2 Fresh cycle characteristics of patients

\begin{tabular}{|c|c|c|c|c|c|}
\hline Characteristics & $\begin{array}{l}\text { Group A } \\
(n=287)\end{array}$ & $\begin{array}{l}\text { Group B } \\
(n=245)\end{array}$ & $\begin{array}{l}\text { Group C } \\
(n=253)\end{array}$ & $\begin{array}{l}\text { Group D } \\
(n=383)\end{array}$ & $p$ value \\
\hline $\mathrm{COC}($ mean $\pm \mathrm{SD})$ & $13.07 \pm 8.21$ & $14.27 \pm 9.36$ & $13.65 \pm 7.52$ & $14.51 \pm 8.16$ & ${ }^{\mathrm{a}} 0.140$ \\
\hline MII (mean \pm SD) & $11.14 \pm 6.59$ & $12.26 \pm 8.05$ & $11.75 \pm 6.28$ & $12.46 \pm 6.96$ & ${ }^{\mathrm{a}} 0.123$ \\
\hline $\mathrm{PN} 2($ mean $\pm \mathrm{SD})$ & $9.40 \pm 5.65$ & $10.19 \pm 6.83$ & $9.7 \pm 5.37$ & $10.45 \pm 5.93$ & ${ }^{\mathrm{a}} 0.145$ \\
\hline \multicolumn{5}{|c|}{ Blastocyst ET morphology } & \multirow[t]{5}{*}{${ }^{\mathrm{b}} 0.893$} \\
\hline Excellent $(n, \%)$ & $176(61.3)$ & $147(60)$ & $150(59.3)$ & $230(60.1)$ & \\
\hline $\operatorname{Good}(n, \%)$ & $90(31.4)$ & $79(32.2)$ & $91(36)$ & $117(30.5)$ & \\
\hline Moderate $(n, \%)$ & $16(5.6)$ & $15(6.1)$ & $9(3.6)$ & $27(7)$ & \\
\hline Poor $(n, \%)$ & $5(1.7)$ & $4(1.6)$ & $3(1.2)$ & $9(2.3)$ & \\
\hline
\end{tabular}

${ }^{a}$ One-way ANOVA

${ }^{\mathrm{b}}$ Pearson chi-square test

$p<0.05$ follicular size but after the confirmation of an LH rise. In cases where the LH rise occurred when the follicular diameter was only $14-15 \mathrm{~mm}$, LH surge was accepted as the main criterion for hCG administration in groups B, C and D. Furthermore, E2 level was simultaneously monitored with LH, but only until hCG day, and E2 drop was not used as a criterion for hCG timing. This was in order to reduce the number of blood samples required from patients.

In Reichmann's study, the LH surge was defined as an LH level > $17 \mathrm{IU} / \mathrm{L}$ during the follicular phase with a subsequent dropping E2 level thereafter. They hypothesized that hCG supplementation after the LH surge would support endogenous steroidogenesis leading to improved pregnancy outcomes. They concluded that natural cycle FET in which the luteal phase is buttressed with both a single hCG injection after the endogenous LH surge, as well as vaginal progesterone after transfer, are associated with higher clinical success rates with minimal negative impact on the patient experience [6]. Our strategy also resulted in high ongoing pregnancy rates (Table 3). According to our results, provided the follicular diameter and e2 levels are appropriate, hCG can be administered at any time between the start of LH rise $(15 \mathrm{IU} / \mathrm{L})$ and LH peak level (> $40 \mathrm{IU} / \mathrm{L})$, without having a detrimental effect on ongoing pregnancy. Furthermore, there was a lower rate of clinical pregnancy losses $(6.1 \%, 5.6 \%, 6.7 \%, 7.3 \%)$ when compared to our pregnancy losses rate in artificial cycle $(17.2 \%)$ [21].

There have been several studies questioning the use of hCG trigger. Mansour et al. [22] reported that hCG increases endometrial progesterone receptors and its level is positively correlated with the level of trophoblastic tolerance. Weisman et al. [23] reported no differences in terms of FET outcome between spontaneous ovulatory cycle patients and hCGadministered patients in FET.

Three studies report a deleterious effect of hCG triggering on implantation and pregnancy rates in spontaneous LH rise patients [8-10]. Fatemi et al. [10] reported that in cryopreserved-thawed human embryo transfer, true natural cycle is superior to human chorionic gonadotropin-induced natural cycle. This study was terminated early, when a prespecified interim analysis found a significantly higher ongoing pregnancy rate in the spontaneous LH group as compared with the hCG group (31.1\% vs. $14.3 \%$; difference $16.9 \%$, $95 \%$ confidence interval 4.4-28.8\%). However, Montagut et al. [24] criticized this study for a number of reasons. Firstly, some women in the mNC-FET group also had an

Table 3 Pregnancy outcomes of patients

\begin{tabular}{llllll}
\hline & $\begin{array}{l}\text { Group A } \\
(n=287)\end{array}$ & $\begin{array}{l}\text { Group B } \\
(n=245)\end{array}$ & $\begin{array}{l}\text { Group C } \\
(n=253)\end{array}$ & $\begin{array}{l}\text { Group D } \\
(n=383)\end{array}$ & $p$ value \\
\hline Biochemical pregnancies $(n, \%)$ & $214(74.6)$ & $177(72.2)$ & $193(76.3)$ & $289(75.5)$ & ${ }^{\mathrm{b} 0} 0.867$ \\
Clinical pregnancies $(n, \%)$ & $201(70.0)$ & $167(68.2)$ & $180(71.1)$ & $268(70)$ & ${ }^{\mathrm{b}} 0.908$ \\
Ongoing pregnancies $(n, \%)$ & $183(63.8)$ & $155(63.3)$ & $165(65.2)$ & $248(64.8)$ & ${ }^{\mathrm{b}} 0.876$ \\
Biochemical pregnancy losses $(n, \%)$ & $13(6.1)$ & $10(5.6)$ & $13(6.7)$ & $21(7.3)$ & ${ }^{\mathrm{b}} 0.445$ \\
Clinical pregnancy losses $(n, \%)$ & $18(8.9)$ & $12(7.2)$ & $15(8.3)$ & $20(7.5)$ & ${ }^{\mathrm{b}} 0.907$ \\
Total pregnancy losses $(n, \%)$ & $31(14.5)$ & $22(12.4)$ & $28(14.5)$ & $41(14.2)$ & ${ }^{\mathrm{b}} 0.886$ \\
\hline
\end{tabular}

${ }^{\mathrm{b}}$ Pearson chi-square test

$p<0.05$ 
LH rise on the day of hCG administration. Secondly, day 3 embryos were transferred. Furthermore, no luteal phase support was given in the mNC-FET group, which could have had a negative impact on clinical outcome $[25,26]$. Finally, FET was scheduled after an identical time interval, irrespective of tNC or mNC-FET, even though ovulation may occur at a later stage after hCG administration compared to ovulation after spontaneous LH rise [25]. Fatemi et al. [10] concluded that there is emerging evidence that the administration of hCG in the late follicular phase induces a cascade of events in the endometrium, which would have started several days later in the presence of endogenous LH rise and that these events appear to have a negative impact on ongoing pregnancy rate. Using our strategy, the ongoing pregnancy rates were $(63.8 \%$, $63.3 \%, 65.2 \%, 64.8 \%$ ), which are significantly higher than the OPR of $14.3 \%$ in their studies. Furthermore, Reichman et al. [6] reported an OPR of 70\% in a retrospective cohort study of women who underwent natural cycle FET with transfer of a single euploid blastocyst and who received hCG 1 day postLH surge. Thus, Fatemi et al.'s cascade hypothesis of events in the endometrium does not explain the low ongoing pregnancy rates that they reported.

Besides the main advantage of supporting the luteal phase in natural cycle via its effect on the corpus luteum, hCG also triggers ovulation and facilitates the programming of embryo transfer day. It is possible that hCG provides a signal to the endometrium regarding future blastocyst implantation, thus fostering the growth and differentiation of trophoblast cells, and the establishment of placental villous structures [27]. It is known that hCG biosynthesis begins early in embryo development, and higher concentrations are found in the uterine cavity prior to implantation. The exogenous administration of hCG during in vitro fertilization may mimic the local effects of hCG when fertilization occurs naturally [28]. Therefore, it is possible that, rather than being detrimental, as has been reported in some studies, the administration of hCG in mNC-FET may have a similar beneficial effect to endogenous hCG.

The protocol at our clinic includes the administration of vaginal progesterone 2 days after the administration of hCG. The question of whether the administration of progesterone to support the luteal phase is necessary for all patients undergoing $\mathrm{mNC}$-FET is still under debate. However, the majority of studies suggest that administration of vaginal progesterone in the luteal phase appears to increase significantly the clinical pregnancy rate [29-31].

One limitation of the study is that it was a retrospective study of good prognosis patients only. It also included a group for whom our usual protocol regarding the timing of hCG was adjusted for the practical reasons described previously and whose LH levels were measured the day before hCG rather than on the day of hCG.
One of the strengths of our study is that it is a single center study, which decreases differences in practice. Furthermore, it is based on the high number of $1168 \mathrm{mNC}$-FET cycles. The administration of hCG at any time between the start of LH rise $(15 \mathrm{IU} / \mathrm{L})$ and peak level $(>40 \mathrm{IU} / \mathrm{L})$ has a significant practical advantage in clinical practice. In the near future, it is likely that modified natural cycle will be used more widely in FET cycles; thus, prospective studies of patients undergoing FET cycles other than those who have good prognosis are needed to guide best practice.

Open Access This article is licensed under a Creative Commons Attribution 4.0 International License, which permits use, sharing, adaptation, distribution and reproduction in any medium or format, as long as you give appropriate credit to the original author(s) and the source, provide a link to the Creative Commons licence, and indicate if changes were made. The images or other third party material in this article are included in the article's Creative Commons licence, unless indicated otherwise in a credit line to the material. If material is not included in the article's Creative Commons licence and your intended use is not permitted by statutory regulation or exceeds the permitted use, you will need to obtain permission directly from the copyright holder. To view a copy of this licence, visit http://creativecommons.org/licenses/by/4.0/.

\section{References}

1. Wong KM, Mastenbroek S, Repping S. Cryopreservation of human embryos and its contribution to in vitro fertilization success rates. Fertil Steril. 2014;102(1):19-26.

2. Roque M, Lattes K, Serra S, Solà I, Geber S, Carreras R, et al. Fresh embryo transfer versus frozen embryo transfer in in vitro fertilization cycles: a systematic review and meta-analysis. Fertil Steril. 2013;99(1):156-62.

3. Roque M, Valle M, Guimarães F, Sampaio M, Geber S. Freeze-all policy: fresh vs. frozen-thawed embryo transfer. Fertil Steril. 2015;103(5):1190-3.

4. Zhu Q, Chen Q, Wang L, Lu X, Lyu Q, Wang Y, et al. Live birth rates in the first complete IVF cycle among 20687 women using a freeze-all strategy. Hum Reprod. 2018;33(5):924-9.

5. Irani M, Robles A, Gunnala V, Reichman D, Rosenwaks Z. Optimal parameters for determining the LH surge in natural cycle frozen-thawed embryo transfers. J Ovarian Res. 2017;10(1):70.

6. Reichman DE, Stewart CR, Rosenwaks Z. Natural frozen embryo transfer with hCG booster leads to improved cycle outcomes: a retrospective cohort study [published online ahead of print, 2020 Mar 20]. J Assist Reprod Genet. 2020;37:1177-82. https://doi.org/ 10.1007/s10815-020-01740-7.

7. Bartels CB, Ditrio L, Grow DR, O'Sullivan DM, Benadiva CA, Engmann L, et al. The window is wide: flexible timing for vitrified-warmed embryo transfer in natural cycles. Reprod BioMed Online. 2019;39(2):241-8.

8. Litwicka K, Mencacci C, Arrivi C, Varricchio MT, Caragia A, Minasi MG, et al. HCG administration after endogenous LH rise negatively influences pregnancy rate in modified natural cycle for frozen-thawed euploid blastocyst transfer: a pilot study. J Assist Reprod Genet. 2018;35:449-55.

9. Kyrou D, Fatemi HM, Blockeel C, Stoop D, Albuarki H, Verheyen $\mathrm{G}$, et al. Transfer of cryopreserved-thawed embryos in hCG induced natural or clomiphene citrate cycles yields similar live birth 
rates in normo-ovulatory women. J Assist Reprod Genet. 2010;27(12):683.

10. Fatemi HM, Kyrou D, Bourgain C, Van den Abbeel E, Griesinger G, Devroey P. Cryopreserved-thawed human embryo transfer: spontaneous natural cycle is superior to human chorionic gonadotropin-induced natural cycle. Fertil Steril. 2010;94(6): 2054-8.

11. Cantineau AE, Cohlen BJ. The prevalence and influence of luteinizing hormone surges in stimulated cycles combined with intrauterine insemination during a prospective cohort study. Fertil Steril. 2007;88(1):107-12.

12. Cohlen BJ, te Velde ER, van Kooij RJ, Looman CW, Habbema JD. Controlled ovarian hyperstimulation and intrauterine insemination for treating male subfertility: a controlled study. Hum Reprod. 1998;13(6):1553-8.

13. Cunha-Filho JS, CKadoch J, Righini C, Fanchin R, Frydman R, Olivennes F. Prematur LH and progesterone rise in intrauterine insemination cycles: analysis of related factors. Reprod BioMed Online. 2003;7(2):194-9.

14. Gardner DK, Lane M, Stevens J, Schlenker T, Schoolcraft WB. Blastocyst score affects implantation and pregnancy outcome: towards single blastocysts transfer. Fertil Steril. 2000;73(6):1155-8.

15. Corroenne R, El Hachem H, Verhaeghe C, Legendre G, Dreux C, Jeanneteau $\mathrm{P}$, et al. Endometrial preparation for frozen-thawed embryo transfer in an artificial cycle: transdermal versus vaginal estrogen. Sci Rep. 2020;10(1):985.

16. Agha Hosseini KM. Natural cycle versus artificial cycle in frozen thawed embryo transfer: a randomized prospective trial. Turk $\mathrm{J}$ Obstet Gynecol. 2018;15:12-7.

17. Groenewoud ER, Kollen BJ, Macklon NS, Cohlen BJ. Spontaneous LH surges prior to HCG administration in unstimulated-cycle frozen-thawed embryo transfer do not influence pregnancy rates. Reprod BioMed Online. 2012;24(2):191-6.

18. Lee VC, Li RH, Chai J, Yeung TW, Yeung WS, Ho PC, et al. Effect of preovulatory progesterone elevation and duration of progesterone elevation on the pregnancy rate of frozen-thawed embryo transfer in natural cycles. Fertil Steril. 2014;101(5):1288-93.

19. Casper RF, Yanushpolsky EH. Optimal endometrial preparation for frozen embryo transfer cycles: window of implantation and progesterone support. Fertil Steril. 2016;105(4):867-72.

20. Saupstad M, Freiesleben NC, Skouby SO, et al. Preparation of the endometrium and timing of blastocyst transfer in modified natural cycle frozen-thawed embryo transfers (mNC-FET): a study protocol for a randomised controlled multicentre trial. BMJ Open. 2019;9(12):e031811.

21. Kahraman S, Çetinkaya CP, Sahin Y, Oner G. Transdermal versus oral estrogen: clinical outcomes in patients undergoing frozen- thawed single blastocyst transfer cycles without GnRHa suppression, a prospective randomized clinical trial. J Assist Reprod Genet. 2019;36(3):453-9.

22. Mansour R, Tawab N, Kamal O, El-Faissal Y, Serour A, Aboulghar $\mathrm{M}$, et al. Intrauterine injection of human chorionic gonadotropin before embryo transfer significantly improves the implantation and pregnancy rates in in vitro fertilization/intracytoplasmic sperm injection: a prospective randomized study. Fertil Steril. 2011;96(6): 1370.e1-1374.

23. Weissman A, Horowitz E, Ravhon A, Steinfeld Z, Mutzafi R, Golan A, et al. Spontaneous ovulation versus HCG triggering for timing natural-cycle frozen-thawed embryo transfer: a randomized study. Reprod BioMed Online. 2011;23(4):484-9.

24. Montagut M, Santos Ribeiro S, De Vos M, et al. Frozen-thawed embryo transfers in natural cycles with spontaneous or induced ovulation: the search for the best protocol continues. Hum Reprod. 2016;31:2803-10.

25. Hill MJ, Whitcomb BW, Lewis TD, Wu M, Terry N, DeCherney $\mathrm{AH}$, et al. Progesterone luteal support after ovulation induction and intrauterine insemination: a systematic review and meta-analysis. Fertil Steril. 2013;100(5):1373-80.

26. Miralpeix E, González-Comadran M, Solà I, Manau D, Carreras R, Checa MA. Efficacy of luteal phase support with vaginal progesterone in intrauterine insemination: a systematic review and metaanalysis. J Assist Reprod Genet. 2014;31(1):89-100.

27. Cole L. Biological functions of hCG and hCG-related molecules. Reprod Biol Endocrinol. 2010;8:102.

28. Licht P, Fluhr H, Neuwinger J, Wallwiener D, Wildt L. Is human chorionic gonadotropin directly involved in the regulation of human implantation? Mol Cell Endocrinol. 2007;269(1-2):85-92.

29. Wang Y, He Y, Zhao X, Ji X, Hong Y, Wang Y, et al. Crinone gel for luteal phase support in frozen-thawed embryo transfer cycles: a prospective randomized clinical trial in the Chinese population. PLoS One. 2015;10(7):e0133027.

30. Zarei A, Sohail P, Parsanezhad ME, Alborzi S, Samsami A, Azizi M. Comparison of four protocols for luteal phase support in frozenthawed embryo transfer cycles: a randomized clinical trial. Arch Gynecol Obstet. 2017;295:239.

31. Schwartz E, Bernard L, Ohl J, Bettahar K, Rongières C, Lichtblau I, et al. Luteal phase progesterone supplementation following induced natural cycle frozen embryo transfer: a retrospective cohort study. J Gynecol Obstet Hum Reprod. 2019;48:95-8.

Publisher's note Springer Nature remains neutral with regard to jurisdictional claims in published maps and institutional affiliations. 